\title{
Robotic-assisted laparoscopic pyeloplasty as management for recurrent ureteropelvic junction obstruction: a comparison study with primary pyeloplasty
}

\author{
Peng Zhang ${ }^{1 \#}$, Taoping Shi ${ }^{1 \#}$, Xenginn Fam ${ }^{2}$, Liangyou Gu ${ }^{1}$, Yundong Xuan ${ }^{1}$, Luojia Yang ${ }^{1}$, \\ Baojun Wang ${ }^{1}$, Xing Ai ${ }^{3}$, Zhuomin Jia ${ }^{3}$, Hongzhao $\mathrm{Li}^{1}$, Xu Zhang ${ }^{1}$, Xin $\mathrm{Ma}^{1}$ \\ ${ }^{1}$ Department of Urology/State Key Laboratory of Kidney Diseases, Chinese PLA General Hospital/Medical School of Chinese PLA, Beijing 100853, \\ China; ${ }^{2}$ Urology Unit, Department of Surgery, UKM Medical Centre, Kuala Lumpur, Malaysia; ${ }^{3}$ Department of Urology, PLA Army General \\ Hospital, Beijing 100853, China \\ Contributions: (I) Conception and design: P Zhang, T Shi, H Li, X Ma, X Zhang; (II) Administrative support: X Zhang; (III) Provision of study \\ material or patients: B Wang, X Ai, H Li, X Ma; (IV) Collection and assembly of data: P Zhang, L Gu, Y Xuan, L Yang, Z Jia; (V) Data analysis and \\ interpretation: P Zhang, X Fam, L Gu; (VI) Manuscript writing: All authors; (VII) Final approval of manuscript: All authors. \\ \#These authors contributed equally to this work. \\ Correspondence to: Xin Ma; Xu Zhang; Hongzhao Li. Prof., Department of Urology/State Key Laboratory of Kidney Diseases, Chinese PLA General Hospital/ \\ Medical School of Chinese PLA, 28 Fuxing Road, Beijing 100853, China. Email: urologist@foxmail.com; xzhang@foxmail.com; urolancet@126.com.
}

Background: To analyze the perioperative parameters and outcomes of robotic-assisted laparoscopic pyeloplasty (RALP) for recurrent ureteropelvic junction obstruction (UPJO) and compare them with our series of RALP for primary UPJO. Secondary pyeloplasty can be a challenging procedure because of ureteral devascularization, fibrosis and dense stricture formation. Robotic approach could be adjunct to these repairs. Methods: Between August 2015 to March 2019, 96 patients in our hospital underwent RALP, with 32 patients as secondary intervention for recurrent UPJO. We compared the perioperative parameters of RALP for both primary UPJO and recurrent UPJO. Patient demographics, perioperative parameters, postoperative outcomes and complications from both groups were analyzed and compared.

Results: RALP was successfully performed for all cases in both groups. The median operating time was longer for secondary RALP than for primary RALP [125 (108.5-155) vs. 151 (120-190) minutes, P=0.004]. There were no conversions to open surgery or significant perioperative complications. No difference in blood loss, transfusion rate and perioperative complication rates was noted between the two groups. The success rates were $98.44 \%(63 / 64)$ and $96.88 \%(31 / 32)$ at a median follow up of 32 and 20 months $(\mathrm{P}=0.001)$ for the primary and secondary groups, respectively.

Conclusions: Secondary RALP is associated with significantly longer operative time as compared to primary RALP, especially during the exposure of the UPJO, however it is a safe surgical modality for recurrent UPJO with durable outcome. RALP should be an alternative treatment modality for recurrent UPJO whenever the facility and expert are available.

Keywords: Robotic-assisted laparoscopic pyeloplasty (RALP); treatment failure; secondary ureteropelvic junction obstruction (secondary UPJO)

Submitted Nov 02, 2019. Accepted for publication Feb 28, 2020.

doi: $10.21037 /$ tau. 2020.03 .25

View this article at: http://dx.doi.org/10.21037/tau.2020.03.25 


\section{Introduction}

Ureteropelvic junction obstruction (UPJO) is one of the commonest cause of hydronephrosis resulted from both congenital and acquired etiology. Pyeloplasty, which was first described by Kuster and thereafter popularized by Anderson and Hynes, remains as the gold standard management of UPJO (1). The success rate of pyeloplasty was reported greater than $94 \%$ across open, laparoscopic and robotic-assisted approaches recently (2-5). However, treatment is not standardized for failed cases that require additional interventions. Secondary repair of UPJO is a very challenging reconstructive procedure due fibrosis surrounding the targeted structure. Literatures describing salvage treatments for recurrent UPJO are limited (6-8).

The clinical feasibility of robotic-assisted laparoscopic pyeloplasty (RALP) has been widely recognized. With its three-dimension magnified camera and multidirection angulation EndoWrist system, robotic surgery system provides great advantage in reconstructive surgery and salvage intervention (9).

As a challenging reconstructive endeavor, Hemal et al. suggested that robotic assistance could minimize the difficulties encountered by laparoscopic approach in managing recurrent cases (10). Literatures that reported the application of robotic surgical system in salvaging recurrent UPJO with their long-term results are very limited. Here, we present our experience with RALP in patients who had failed prior surgical management of UPJO and report our mid-term outcomes.

\section{Methods}

\section{Study design}

This is a retrospective study. From August 2015 until March 2019, 96 patients in our hospital underwent robotic dismembered RALP using the da Vinci Surgical System. Among these patients, RALP was carried out in 32 patients as secondary intervention for recurrent UPJO. The baseline demographic parameters of these patients were recorded as Table 1 .

Indications for RALP included flank pain, recurrent febrile infection, stone, and progression of hydronephrosis. Intravenous urography (IVU) or retrograde pyelography, CT or MRI scan, radioisotope renography were carried out preoperatively to assess the anatomy of ureteropelvic junction and surrounding structures, degree of obstruction and split renal function.

\section{Surgical technique}

All surgeries were performed by two experienced surgeons (Xu Zhang and Xin Ma) using Intuitive Surgical da Vinci Si system. Preoperative double-J ureteral stent was inserted in 13 patients ( 5 in primary RALP cohort and 8 in secondary RALP cohort) and nephrostomy tube was placed in 6 patients ( 4 in primary RALP cohort and 2

Table 1 Patient demographics

\begin{tabular}{|c|c|c|c|}
\hline Characteristics & Primary RALP $(n=64)$ & Secondary RALP $(n=32)$ & $P$ value \\
\hline $\operatorname{Sex}(n)$ & & & 0.209 \\
\hline Male & 42 & 25 & \\
\hline Female & 22 & 7 & \\
\hline Laterality (n) & & & 0.753 \\
\hline Left & 44 & 23 & \\
\hline Right & 20 & 9 & \\
\hline Preoperative symptoms & $35 / 64$ & $14 / 32$ & 0.312 \\
\hline
\end{tabular}

RALP, robot-assisted laparoscopic pyeloplasty; BMI, body mass index; SD, standard deviation; IQR, interquartile range. 

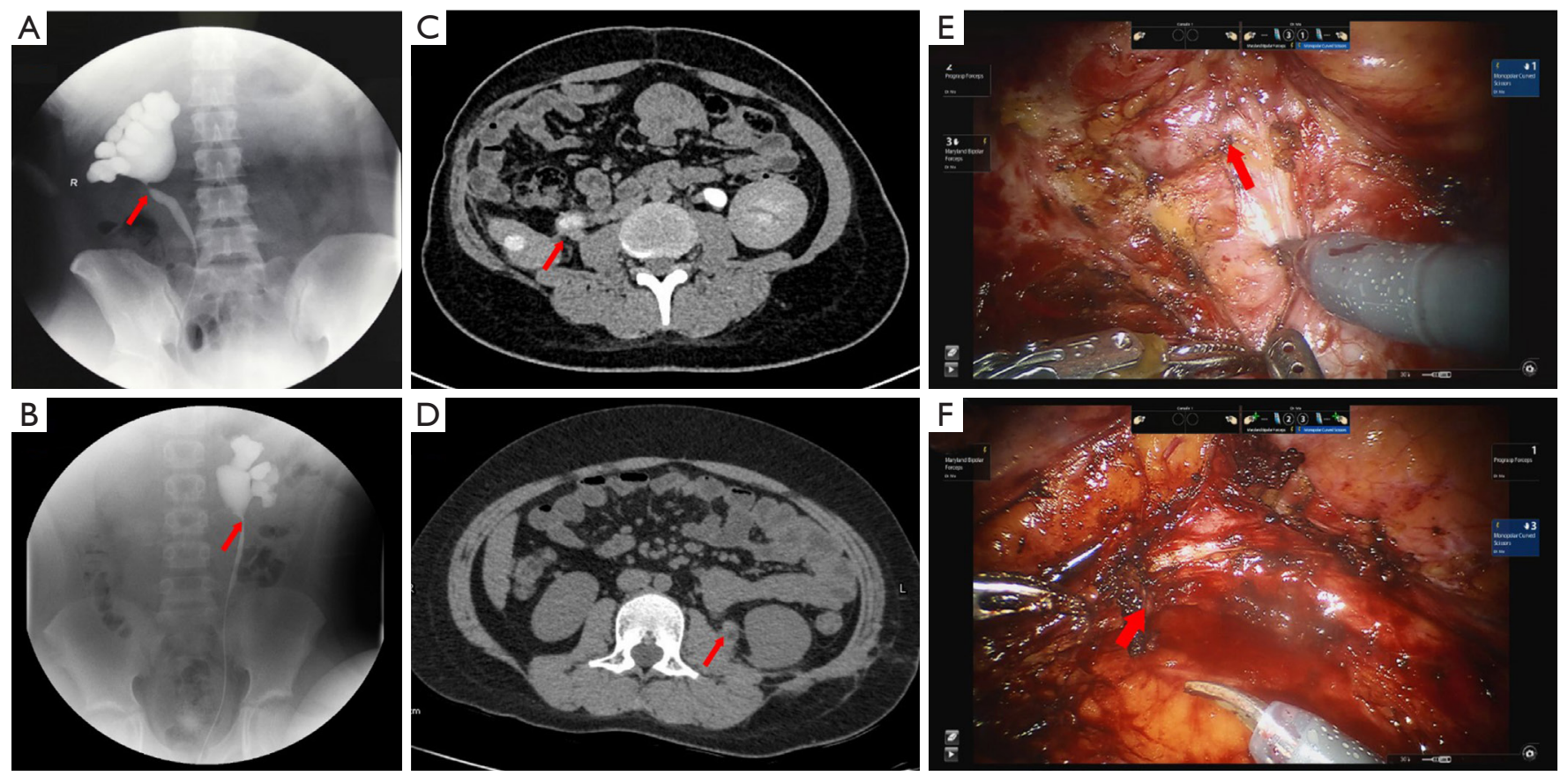

Figure 1 UPJ stenosis and fibrosis. (A,B) Retrograde pyelogram showed stenosis at the uretero-pelvis junction; (C,D) axial CT scan images showed UPJ stenosis with surrounding scar and adhesion; (E,F) intraoperative images showed dense scar and adhesion around the area of UPJ stenosis. UPJ, ureteropelvic junction.

in secondary RALP cohort) due to severe flank pain or infected hydronephrosis. All operations were performed transperitoneally with standard 3-arm technique and one assistant port. Modified dismembered Anderson-Hynes pyeloplasty technique was performed for all cases. For secondary RALP, the standard dissection plane was difficult to be identified due to fibrosis (Figure 1); we started the dissection from healthy ureter segment with minimal adhesion and mobilized proximally until renal pelvis. Stricture was completely resected with minimal injury to ureter mucosa; subsequently modified dismembered pyeloplasty was performed. To assure a tension-free anastomosis, the kidney might be mobilized as indicated. The double-J stent was inserted antegradely (Video 1). Anterior crossing vessels were preserved and transposed posteriorly whenever encountered. An abdominal drainage was placed conventionally and removed $2-3$ days after surgery when the possibility of urine leakage was eliminated. Ureteral stent was removed 1 month postoperatively.

\section{Measurements and outcomes}

Perioperative data including median operative time, estimated blood loss, postoperative hospital stay, interval from the previous operation and perioperative complications were assessed. Complications were categorized according to the Clavien-Dindo classification system. Patient was follow-up every 3 months during the first year and every 6 months during the second year. The follow-up assessments included physical examination, full blood count and renal profile. Abdominal ultrasonography, CT or MRI scan and renal scans were performed at 3,6 months and then annually. Successful operation was defined as combination of symptomatic relief, radiographic evidence of ureteral patency, first postoperative radioisotope renography showed split function improvement with a half-time less than 20 minutes after the diuretic or pelvic dilatation improvement during excretory phase, and stable or improved renal function without any additional procedure postoperatively, for at least 6 months of follow-up.

\section{Statistical analyses}

All mentioned parameters were maintained in database. Parameters for primary and secondary repairs were compared and analyzed. Perioperative and postoperative 
outcomes were reported. For continuous variables with normal distribution, data were presented as mean with standard deviation (SD); for variables with nonnormal distribution, data were presented as median with interquartile range (IQR). All statistical analyses were performed using SPSS statistical software package (SPSS Inc., Chicago, IL, USA).

The study was approved by the ethics committee of the Chinese PLA General Hospital (S201311501). All patients signed written consent to allow the usage of their data. All operations were performed by surgeons with advanced robotic-assisted laparoscopic skills.

\section{Results}

Comparison of patients underwent primary RALP and secondary RALP was listed in Table 1. There was no significance different in age, gender and laterality. The mean interval duration from previous failed pyeloplasty was 66 (17.5-156, IQR) months. Thirteen in secondary RALP group patients presented with flank pain, among these patients, 2 associated with fever, 1 with nausea, 1 with stone and 1 with hydronephrosis. 1 patient presented with fever due to urinary tract infection. Asymptomatic progressing of hydronephrosis and asymptomatic renal calculus were observed in 15 and 3 patients respectively. Initial procedures were performed for patients in secondary RALP group included pyelolithotomy, open, laparoscopic and robotic pyeloplasty (Table 2). As compared to secondary RALP, primary RALP group had a longer follow-up duration [32 (22-36) vs. 20 (13-24) months, $\mathrm{P}=0.001$ ] (Table 3).

RALP was successfully performed for all cases in both groups; neither of them converted to open surgery nor required blood transfusion. As compared to primary RALP, secondary RALP was associated with longer operative time [125 (108.75-155) vs. 151 (120-190) minutes, $\mathrm{P}=0.004]$ and shorter postoperative hospital stay [5 (4-6.255) vs. 4 (3-5) days, $\mathrm{P}=0.008]$. Difference in estimated blood loss was not significant (Table 3).

There was no intraoperative complication in primary RALP group; whereas, one intraoperative complications complication was observed in secondary RALP group. Renal vein was injured in one patient due to dense fibrosis and was repaired intracorporeally. Postoperative complications were observed in both groups. In primary RALP cohort, two patients presented with urinary leakage, and resolved spontaneously after a prolonged duration of the drainage. Frank hematuria was identified in two patients, and subsequently resolved with conservative management. One patient presented with fever and required anti-inflammatory treatment. One patient was complicated with wound infection, and treated with antibiotic. In secondary RALP cohort, one patient was complicated with urinary leakage and resolved with prolonged drainage. Frank hematuria was identified in another patient and resolved with conservative management. The incidence of complications between primary RALP (6 of 64) and secondary RALP (2 of 32) $(\mathrm{P}=0.896)$ was not statistically significant. There was one patient from each cohort showed failure of treatment as evidence by non-improvement of hydronephrosis in primary RALP cohort; and worsening of hydronephrosis in secondary RALP cohort. Success rates were $98.44 \%$ and $96.88 \%$ for the primary and secondary groups, respectively $(\mathrm{P}=1.000)$ (Table 3).

\section{Discussion}

As described in most literature, salvage treatments for recurrent UPJO patients were open pyeloplasty, endoscopic pyelotomy or balloon dilatation (11-13). However, open approach is traumatizing, and lead to slower recovery. Open surgery induces tissue adhesion and fibrosis that might contribute to subsequent recurrent of UPJO. The success rate of endoscopic pyelotomy and balloon dilatation is low as compared to salvage anastomosis operation $(13,14)$. In recent years, minimal invasive surgery, especially robotic surgery progresses rapidly, which provides an alternative option for recurrent UPJO patients who had failed prior surgical management.

Several studies had confirmed the feasibility of secondary laparoscopic pyeloplasty (LP) for recurrent UPJO (15). The success rate of redo LP was $77.8-100 \%$ (16-18). However, secondary LP for recurrent UPJO was very challenging, and skill demanding. Presence of significant periureteral fibrosis demands extra time for delineation of the UPJ anatomy, dissection of scarred tissue, fashioning ureteral and pelvic flaps clearly, kidney mobilization in some cases and finally, performing watertight anastomosis with fine sutures. All mentioned operative steps increase difficulty of the operation, significantly prolong operative time and associate with higher complication rates in comparison with primary UPJO (19). Thus, secondary LP in recurrent UPJO has been limited to be performed in a high volume of centers due to its difficulty.

Over the past decades, robotic-assisted surgeries have been expanded, and applied its usage in complicated 
Table 2 Patient characteristics at operation and reoperation

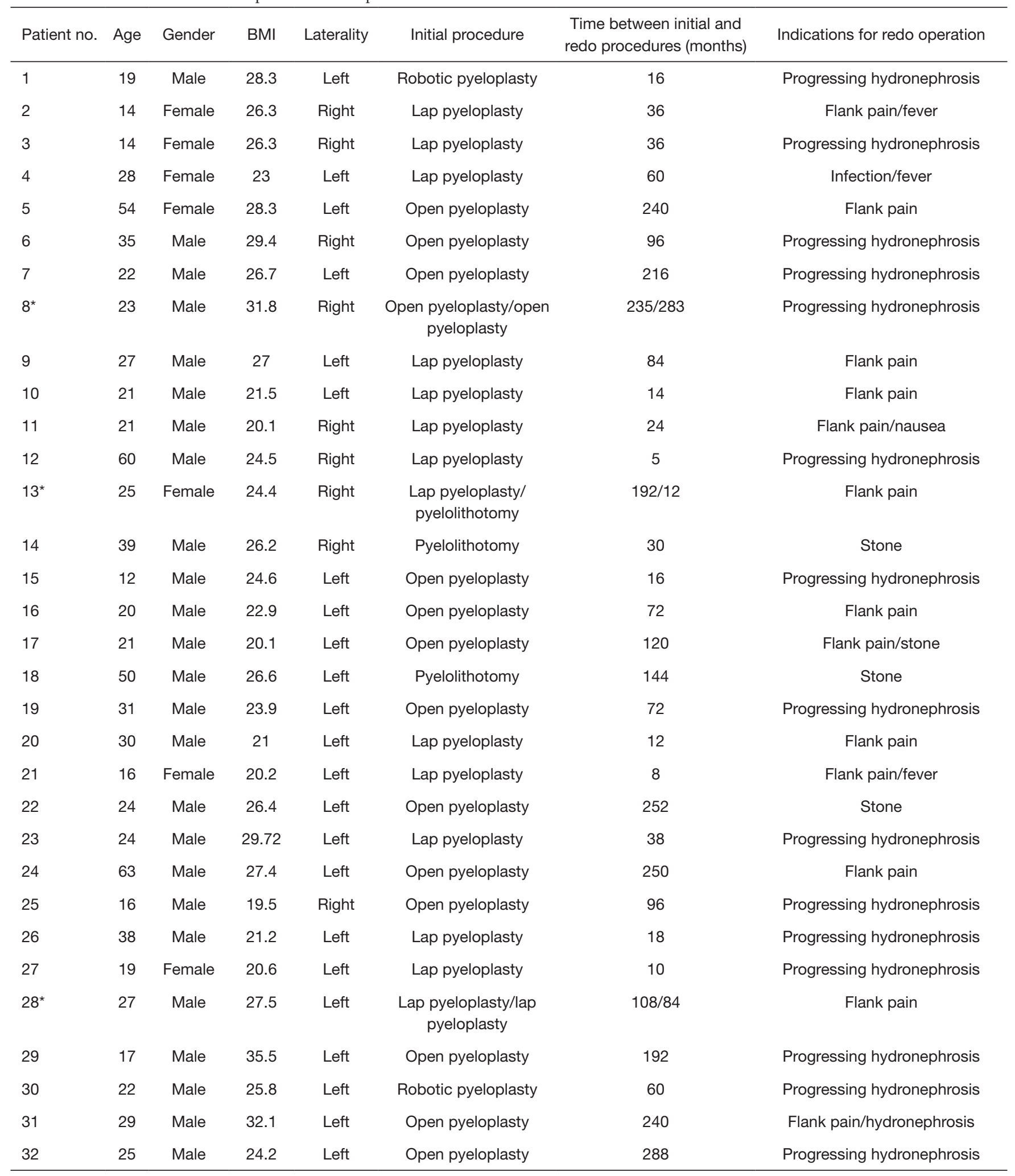

*, Patients with third-time redo pyeloplasty. BMI, body mass index. 
Table 3 Operative and postoperative outcomes

\begin{tabular}{|c|c|c|c|}
\hline Characteristics & Primary RALP $(n=64)$ & Secondary RALP $(n=32)$ & $P$ value \\
\hline Estimated blood loss, $\mathrm{mL}$, median [range] & 20 [5-200] & $50[10-100]$ & 0.119 \\
\hline Transfusion rate, $\mathrm{n}(\%)$ & 0 & 0 & $\mathrm{~N}$ \\
\hline Intraoperative conversion rate & 0 & 0 & $\mathrm{~N}$ \\
\hline Stones present $(n)$ & $9 / 64$ & $4 / 32$ & 0.9625 \\
\hline Postoperative hospital stay, median [IQR] & $5[4-6.25]$ & $4[3-5]$ & 0.008 \\
\hline Follow up range, month, median [IQR] & 32 [22-36] & 20 [13-24] & 0.001 \\
\hline \multicolumn{4}{|l|}{ Complications } \\
\hline Grade I & 4 & 2 & \\
\hline Grade II & 2 & 0 & \\
\hline Success rate, $\%$ (n/total) & $98.44(63 / 64)$ & $96.88(31 / 32)$ & 1.000 \\
\hline
\end{tabular}

RALP, robot-assisted laparoscopic pyeloplasty; SD, standard deviation; IQR, interquartile range.

surgeries. The advantages of da Vinci robot surgery, which are its three-dimension magnified vision and multiangle angulation EndoWrist system have been widely recognized. Third robotic arm that is very useful in tissue retraction is crucial in such kind of complex urinary tract reconstruction surgery $(20,21)$. Robotic approach provide the benefits of meticulous dissection, better delineation of the previous scarred tissue, preservation of the periureteral sheath containing blood supply to the ureter, clean and fine fashioning of ureteral and pelvic flaps and executing water tight anastomosis with fine sutures. Several publication had confirmed the safety and feasibility of RALP (22-24). Gettman et al., in 2002, reported the first case series of robotic pyeloplasty (9). Atug et al. then reported on seven adult patients with secondary RALP and compared their results with 37 primary UPJO patients (25). The mean operative time was longer in the redo pyeloplasty group, but the LOS, blood loss and success rates were similar with the primary pyeloplasty group. Hemal et al. reported nine patients underwent secondary RALP after failed open pyeloplasty with ideal improvement (10). Niver et al. showed safe and durable results in 20 cases of redo robotassisted pyeloplasty in adults (26). The safety and effective of robot-assisted laparoscopic reoperative repair of UPJO have also been published in children by Lindgren $e t$ al. with
$88 \%$ radiographically improvement (27).

In our study, the mean operative time was longer in the secondary RALP group. Extra time was required in redo RALP to dissect scarred and fibrotic tissue for identifying standard dissection plan. However, the blood loss, transfusion rate, incidence of perioperative complications and success rates were similar in both primary RALP and secondary RALP groups. Patients with postoperative complications including urinary leakage, frank hematuria and wound infection were managed conservatively as inpatient for longer duration that contributed to the longer postoperative hospital stay of primary RALP cohort. Generally, the redo RALP was comparable with primary RALP in safety and effective.

We identified a series of maneuvers to overcome the challenges of secondary repair for recurrent UPJO. Transperitoneal approach was recommended, as this approach has more anatomical landmarks and larger surgical space, which is useful during dissection of fibrotic tissue. Dissection should be started from normal ureter segment with minimal adhesion, where the standard dissection plane can be easily identified. Periureteral fascia must be preserved during dissection, as it is the blood supply to ureter. Ureter mucosa must be handled with care to avoid injury, which can contribute to fibrosis in long term. A 
tension-free anastomosis is crucial for good outcome of the operation. Ureter must be fully mobilized and kidneys should be mobilized as required. The interval from previous failed pyeloplasty was recommended to be more than 1 year for patients with stable renal function. Short interval from previous operation may associate with severe tissue edema or adhesion.

There are several limitations in our article. First, this was a single institutional retrospective study. In the future, more cases from other centers could be enrolled to strength our conclusion. Second, this was a retrospect study with more than 6 months follow up, therefore, other prospective study with longer period of follow up are required to validate our results. Third, some patients neglected renal scan examination due to symptomatic improvement postoperatively and remission of hydronephrosis. So, in future research, close follow-up and patient education should be strengthened.

\section{Conclusions}

The management of recurrent UPJO is technically challenging. The low success rates of endourological procedures had increased the role of redo pyeloplasty in such patients. Robot-assisted redo pyeloplasty had proven its feasibility and offered an equivalent success rate as primary RALP. However, secondary RALP is associated with significantly longer operative time as compared with primary RALP, especially during the exposure of the UPJ. Series of evidences strongly suggest that RALP is a safe and durable option for secondary UPJO repair. We suggest that RALP should be an alternative treatment modality for recurrent UPJO whenever the facility and expert are available.

\section{Acknowledgments}

Funding: None.

\section{Footnote}

Conflicts of Interest: All authors have completed the ICMJE uniform disclosure form (available at http://dx.doi. org/10.21037/tau.2020.03.25). The authors have no conflicts of interest to declare.

Ethical Statement: The authors are accountable for all aspects of the work in ensuring that questions related to the accuracy or integrity of any part of the work are appropriately investigated and resolved. The study was approved by the ethics committee of the Chinese PLA General Hospital (S201311501). All patients signed written consent to allow the usage of their data. All operations were performed by surgeons with advanced robotic-assisted laparoscopic skills.

Open Access Statement: This is an Open Access article distributed in accordance with the Creative Commons Attribution-NonCommercial-NoDerivs 4.0 International License (CC BY-NC-ND 4.0), which permits the noncommercial replication and distribution of the article with the strict proviso that no changes or edits are made and the original work is properly cited (including links to both the formal publication through the relevant DOI and the license). See: https://creativecommons.org/licenses/by-nc-nd/4.0/.

\section{References}

1. Anderson JC, Hynes W. Retrocaval ureter; a case diagnosed pre-operatively and treated successfully by a plastic operation. Br J Urol 1949;21:209-14.

2. Göğüş C, Karamürsel T, Tokatli Z, et al. Long-term results of Anderson-Hynes pyeloplasty in 180 adults in the era of endourologic procedures. Urol Int 2004;73:11-4.

3. Romero FR, Wagner AA, Trapp C, et al. Transmesenteric laparoscopic pyeloplasty. J Urol 2006;176:2526-9.

4. Thom MR, Haseebuddin M, Roytman TM, et al. Robotassisted pyeloplasty: outcomes for primary and secondary repairs, a single institution experience. Int Braz J Urol 2012;38:77-83.

5. Masieri L, Sforza S, Mari A, et al. Robot-assisted pyeloplasty for ureteropelvic junction obstruction: experience from a tertiary referral center. Minerva Urol Nefrol 2019;71:168-73.

6. Piaggio LA, Noh PH, González R. Reoperative laparoscopic pyeloplasty in children: comparison with open surgery. J Urol 2007;177:1878-82.

7. Braga LH, Lorenzo AJ, Skeldon S, et al. Failed pyeloplasty in children: comparative analysis of retrograde endopyelotomy versus redo pyeloplasty. J Urol 2007;178:2571-5; discussion 2575.

8. Dy GW, Hsi RS, Holt SK, et al. National trends in secondary procedures following pediatric pyeloplasty. J Urol 2016;195:1209-14.

9. Gettman MT, Neururer R, Bartsch G, et al. AndersonHynes dismembered pyeloplasty performed using the da 
Vinci robotic system. Urology 2002;60:509-13.

10. Hemal AK, Mishra S, Mukharjee S, et al. Robot assisted laparoscopic pyeloplasty in patients of ureteropelvic junction obstruction with previously failed open surgical repair. Int J Urol 2008;15:744-6.

11. Thomas JC, DeMarco RT, Donohoe JM, et al. Management of the failed pyeloplasty: a contemporary review. J Urol 2005;174:2363-6.

12. Motola JA, Badlani GH, Smith AD. Results of 212 consecutive endopyelotomies: an 8-year followup. J Urol 1993;149:453-6.

13. Preminger GM, Clayman RV, Nakada SY, et al. A multicenter clinical trial investigating the use of a fluoroscopically controlled cutting balloon catheter for the management of ureteral and ureteropelvic junction obstruction. J Urol 1997;157:1625-9.

14. Dimarco DS, Gettman MT, McGee SM, et al. Longterm success of antegrade endopyelotomy compared with pyeloplasty at a single institution. J Endourol 2006;20:707-12.

15. Abraham GP, Siddaiah AT, Ramaswami K, et al. Laparoscopic management of recurrent ureteropelvic junction obstruction following pyeloplasty. Urol Ann 2015;7:183-7.

16. Basiri A, Behjati S, Zand S, et al. Laparoscopic pyeloplasty in secondary ureteropelvic junction obstruction after failed open surgery. J Endourol 2007;21:1045-51; discussion 1051.

17. Nakada SY, McDougall EM, Clayman RV. Laparoscopic pyeloplasty for secondary ureteropelvic junction obstruction: preliminary experience. Urology 1995;46:257-60.

18. Nishi M, Tsuchida M, Ikeda M, et al. Laparoscopic pyeloplasty for secondary ureteropelvic junction obstruction: long-term results. Int J Urol 2015;22:368-71.
19. Hammady A, Elbadry MS, Rashed EN, et al. Laparoscopic repyeloplasty after failed open repair of ureteropelvic junction obstruction: a case-matched multi-institutional study. Scand J Urol 2017;51:402-6.

20. Arlen AM, Broderick KM, Travers C, et al. Outcomes of complex robot-assisted extravesical ureteral reimplantation in the pediatric population. J Pediatr Urol 2016;12:169.e1-6.

21. Masieri L, Sforza S, Di Maida F, et al. Robotic correction of iatrogenic ureteral stricture: preliminary experience from a tertiary referral centre. Scand J Urol 2019;53:356-60.

22. Gettman MT, Peschel R, Neururer R, et al. A comparison of laparoscopic pyeloplasty performed with the daVinci robotic system versus standard laparoscopic techniques: initial clinical results. Eur Urol 2002;42:453-7; discussion 457-8.

23. Mendez-Torres F, Woods M, Thomas R. Technical modifications for robot-assisted laparoscopic pyeloplasty. J Endourol 2005;19:393-6.

24. Hopf HL, Bahler CD, Sundaram CP. Long-term outcomes of robot-assisted laparoscopic pyeloplasty for ureteropelvic junction obstruction. Urology 2016;90:106-10.

25. Atug F, Burgess SV, Castle EP, et al. Role of robotics in the management of secondary ureteropelvic junction obstruction. Int J Clin Pract 2006;60:9-11.

26. Niver BE, Agalliu I, Bareket R, et al. Analysis of roboticassisted laparoscopic pyleloplasty for primary versus secondary repair in 119 consecutive cases. Urology 2012;79:689-94.

27. Lindgren BW, Hagerty J, Meyer T, et al. Robot-assisted laparoscopic reoperative repair for failed pyeloplasty in children: a safe and highly effective treatment option. J Urol 2012;188:932-7.
Cite this article as: Zhang P, Shi T, Fam X, Gu L, Xuan Y, Yang L, Wang B, Ai X, Jia Z, Li H, Zhang X, Ma X. Roboticassisted laparoscopic pyeloplasty as management for recurrent ureteropelvic junction obstruction: a comparison study with primary pyeloplasty. Transl Androl Urol 2020;9(3):1278-1285. doi:10.21037/tau.2020.03.25 